Appl. Set-Valued Anal. Optim. 3 (2021), No. 1, pp. 27-37

Available online at http://asvao.biemdas.com

https://doi.org/10.23952/asvao.3.2021.1.04

\title{
APPROXIMATIONS METHOD FOR FIXED POINTS OF FURTHER 2-GENERALIZED HYBRID MAPPINGS IN FLAT HADAMARD SPACES
}

\author{
BASHIR ALI ${ }^{1, *}$, LAWAL YUSUF HARUNA ${ }^{2}$ \\ ${ }^{1}$ Department of Mathematical Sciences, Bayero University, Kano, Nigeria \\ ${ }^{2}$ Department of Mathematical Sciences, Kaduna State University, Kaduna, Nigeria
}

\begin{abstract}
In this paper, a hybrid iterative algorithm for approximating fixed points of noncommutative further 2-generalized hybrid mappings is proposed. We prove that the sequence generated by the proposed algorithm converges strongly to a common fixed point of such mappings in flat Hadamard spaces. The result established in this paper generalizes and improves some recently results announced in the literature.
\end{abstract}

Keywords. Further generalized hybrid mapping; Normally 2-generalized hybrid mapping; Flat Hadamard space; Further 2-generalized hybrid mapping.

\section{INTRODUCTION}

Let $H$ be a real Hilbert space, and let $C$ be a nonempty subset of $H$. Let $T: C \rightarrow H$ be a nonlinear mapping. We denote the set of fixed points of $T$ by $F(T)$, i.e., $F(T)=\{u \in C: T u=$ $u$ \}. A mapping $T: C \rightarrow H$ is said to be $(\alpha, \beta)$-generalized hybrid [1] if there exist $\alpha, \beta \in \mathbb{R}$ such that

$$
\alpha\|T x-T y\|^{2}+(1-\alpha)\|x-T y\|^{2} \leq \beta\|T x-y\|^{2}+(1-\beta)\|x-y\|^{2} \forall x, y \in C .
$$

Observe that the mapping $T$ is reduced to a nonexpansive mapping if $\alpha=1$ and $\beta=0$, i.e., $\|T x-T y\| \leq\|x-y\|, \forall x, y \in C$. If $\alpha=\frac{3}{2}$ and $\beta=\frac{1}{2}$, then $T$ is said to be hybrid [2, 3], i.e.,

$$
3\|T x-T y\|^{2} \leq\|x-y\|^{2}+\|T x-y\|^{2}+\|T y-x\|^{2}, \quad \forall x, y \in C .
$$

Also, it is called nonspreading [4], i.e.,

$$
2\|T x-T y\|^{2} \leq\|T x-y\|^{2}+\|T y-x\|^{2}, \quad \forall x, y \in C,
$$

if $\alpha=2$ and $\beta=1$. Recall that a mapping $T$ is called normally generalized hybrid [5] if there exist $\alpha, \beta, \gamma, \delta \in \mathbb{R}$ such that

(a) $\alpha+\beta+\gamma+\delta \geq 0$; (b) $\alpha+\beta>0$ or $\alpha+\gamma>0$ and

$$
\alpha\|T x-T y\|^{2}+\beta\|x-T y\|^{2}+\gamma\|T x-y\|^{2}+\delta\|x-y\|^{2} \leq 0, \forall x, y \in C .
$$

The class of normally generalized hybrid mapping is a subclass of the generalized hybrid mapping. As a generalization of this class of normally generalized hybrid mapping, the classes of

${ }^{*}$ Corresponding author.

E-mail addresses: bashiralik@yahoo.com (B. Ali), yulah121@gmail.com (L.Y. Haruna).

Received May 9, 2020; Accepted July 17, 2020.

(C)2021 Applied Set-Valued Analysis and Optimization 
normally 2-generalized hybrid and further generalized hybrid mappings were introduced. A mapping $T$ is called

(i) normally 2-generalized hybrid [6] if there exist $\alpha_{1}, \alpha_{2}, \alpha_{3}, \beta_{1}, \beta_{2}, \beta_{3} \in \mathbb{R}$ such that (a) $\sum_{i=1}^{3}\left(\alpha_{i}+\beta_{i}\right) \geq 0$, (b) $\sum_{i=1}^{3} \alpha_{i}>0$ and

$$
\begin{aligned}
& \alpha_{1}\left\|T^{2} x-T y\right\|^{2}+\alpha_{2}\|T x-T y\|^{2}+\alpha_{3}\|x-T y\|^{2} \\
& \quad+\beta_{1}\left\|T^{2} x-y\right\|^{2}+\beta_{2}\|T x-y\|^{2}+\beta_{3}\|x-y\|^{2} \leq 0, \forall x, y \in C .
\end{aligned}
$$

(ii) further generalized hybrid [7] if there exist $\alpha, \beta, \gamma, \delta, \varepsilon \in \mathbb{R}$ such that

(a) $\alpha+\beta+\gamma+\delta \geq 0, \varepsilon \geq 0$ (b) $\alpha+\beta>0$ or $\alpha+\gamma>0$ and

$$
\begin{aligned}
\alpha\|T x-T y\|^{2} & +\beta\|x-T y\|^{2}+\gamma\|T x-y\|^{2} \\
& +\delta\|x-y\|^{2}+\varepsilon\|x-T x\|^{2} \leq 0, \forall x, y \in C .
\end{aligned}
$$

In 2019, Takahashi, Wen and Yao [8] proved strong convergence theorems via hybrid methods for two noncommutative normally 2-generalized hybrid mappings in Hilbert spaces. They proved that the sequence $\left\{x_{n}\right\} \subset C$ defined by

$$
\left\{\begin{array}{l}
x_{1}=x \in C \\
y_{n}=a_{n} x_{n}+b_{n}\left(\gamma_{n} S+\left(1-\gamma_{n}\right) T\right) x_{n}+c_{n}\left(\delta_{n} S^{2}+\left(1-\delta_{n}\right) T^{2}\right) x_{n}, \\
C_{n}=\left\{z \in C:\left\|y_{n}-z\right\| \leq\left\|x_{n}-z\right\|\right\} \\
Q_{n}=\left\{z \in C:\left\langle x_{n}-z, x_{1}-x_{n}\right\rangle \geq 0\right\} \\
x_{n+1}=P_{C_{n} \cap Q_{n}} x_{1}, \quad \forall n \in \mathbb{N}
\end{array} .\right.
$$

converges strongly to $z_{0}=P_{F(S) \cap F(T)^{x}}$, where $P_{F(S) \cap F(T)}$ is the metric projection of $H$ on $F(S) \cap F(T)$.

Let $(X, d)$ be a metric space and $x, y \in X$. An isometry $c:[0, d(x, y)] \rightarrow X$ satisfying $c(0)=x$ and $c(d(x, y))=y$ is called a geodesic path joining $x$ to $y$. A geodesic segment between $x$ and $y$ is the image of a geodesic path joining $x$ to $y$, which is denoted by $[x, y]$ provided that it is unique. A geodesic space is a metric space $(X, d)$ in which every two points of $X$ are joined by a geodesic segment. It is said to be a uniquely geodesic space if every two points of $X$ are joined by only one geodesic segment. Let $X$ be a uniquely geodesic space, and let $(1-t) x \oplus t y$ denote the unique point $z$ of the geodesic segment joining $x$ to $y$ for each $x, y \in X$ such that $d(z, x)=t d(x, y)$ and $d(z, y)=(1-t) d(x, y)$. Set $[x, y]:=\{(1-t) x \oplus t y: t \in[0,1]\}$. Then, a subset $C \subset X$ is said to be convex if $[x, y] \subset C$ for all $x, y \in C$.

A geodesic triangle $\Delta\left(x_{1}, x_{2}, x_{3}\right)$ in a geodesic space $(X, d)$ consists of three points in $X$ (the vertices of $\Delta$ ) and a geodesic segment between each pair of points (the edges of $\Delta$ ). A comparison triangle for $\Delta\left(x_{1}, x_{2}, x_{3}\right)$ in $(X, d)$ is a triangle $\bar{\Delta}\left(x_{1}, x_{2}, x_{3}\right)=\Delta\left(\overline{x_{1}}, \overline{x_{2}}, \overline{x_{3}}\right)$ in the Euclidean plane $\mathbb{R}^{2}$ such that $d_{\mathbb{R}^{2}}\left(\bar{x}_{i}, \bar{x}_{j}\right)=d\left(x_{i} . x_{j}\right)$ for all $i, j \in\{1,2,3\}$.

A geodesic space $X$ is called a $C A T(0)$ space if all geodesic triangles of appropriate size satisfy the following comparison axiom: Let $\Delta$ be a geodesic triangle in $X$ and let $\bar{\Delta}$ be a comparison triangle in $\mathbb{R}^{2}$. Then the triangle $\Delta$ is said to satisfy the $C A T(0)$ in equality if $d(x, y) \leq d_{\mathbb{R}^{2}}(\bar{x}, \bar{y})$ for all $x, y \in \Delta$ and all comparison points $\bar{x}, \bar{y} \in \bar{\Delta}$.

Lemma 1.1. [9] Let $X$ be a uniquely geodesic space. The following assertions are equivalent:

(i) $X$ is a $C A T(0)$ space; 
(ii) $X$ satisfies the $C A T(0)(C N)$ inequality, i.e., if $x, y, z$ are points in $X$, and $q$ is the midpoint of the segment $[y, z]$, then

$$
d^{2}(x, q) \leq \frac{1}{2} d^{2}(x, y)+\frac{1}{2} d^{2}(x, z)-\frac{1}{4} d^{2}(y, z) .
$$

A complete CAT(0) space is called a Hadamard space. The examples of Hadamard spaces include Hilbert spaces, the Hilbert ball [10], Euclidean spaces $\mathbb{R}^{n}, \mathbb{R}$-trees, hyperbolic spaces [11] and any complete simply connected Riemanian manifold with non-positive sectional curvatures.

Definition 1.1. [12] A Hadamard space $X$ is called flat if and only if, for each $x, y, z \in X$ and $t \in[0,1], d^{2}((1-t) x \oplus t y, z)=(1-t) d^{2}(x, z)+t d^{2}(y, z)-t(1-t) d^{2}(x, y)$.

It is known [12] that, in a flat Hadamard space $X$, for each $x, y, z, w \in X$ and $t \in[0,1]$,

$$
\langle\overrightarrow{x y}, \overrightarrow{x(t z \oplus(1-t) w)}\rangle=t\langle\overrightarrow{x y}, \overrightarrow{x z}\rangle+(1-t)\langle\overrightarrow{x y}, \overrightarrow{x w}\rangle \text {. }
$$

Let $\left\{x_{n}\right\}$ be a bounded sequence in a complete CAT(0) space $X$ and, for $x \in X, r\left(x,\left\{x_{n}\right\}\right):=$ $\limsup _{n \rightarrow \infty} d\left(x, x_{n}\right)$, the asymptotic radius of $\left\{x_{n}\right\}$ is given by $r\left(\left\{x_{n}\right\}\right)=\inf \left\{r\left(x,\left\{x_{n}\right\}\right): x \in X\right\}$, and the asymptotic center of $\left\{x_{n}\right\}$ is the set $A\left(\left\{x_{n}\right\}\right)=\left\{x \in X: r\left(x,\left\{x_{n}\right\}\right)=r\left(\left\{x_{n}\right\}\right)\right\}$. In a complete CAT(0) sapce, it is generally known that $A\left(\left\{x_{n}\right\}\right)$ consists of exactly one point; see [13]. A sequence $\left\{x_{n}\right\}$ is said to be $\Delta$-convergent to a point $x \in X$ if, for every subsequence $\left\{x_{n_{k}}\right\}$ of $\left\{x_{n}\right\}, A\left(\left\{x_{n_{k}}\right\}\right)=\{x\}$. In this case, $x$ is called $\Delta$-limit of $\left\{x_{n}\right\}$ and it is written as $\Delta-\lim _{n \rightarrow \infty} x_{n}=x$.

In 2008, Berg and Nicolev [14] introduced the concept of the quasilinearisation in a complete CAT( 0$)$ space. They denoted a pair $(a, b) \in X \times X$ by $\overrightarrow{a b}$ and called it a vector. The quasilinearisation is a map $\langle.,\rangle:.(X \times X) \times(X \times X) \rightarrow \mathbb{R}$ defined by

$$
\langle\overrightarrow{a b}, \overrightarrow{c d}\rangle=\frac{1}{2}\left(d^{2}(a, d)+d^{2}(b, c)-d^{2}(a, c)-d^{2}(b, d)\right)
$$

for every $a, b, c, d \in X$. From the definition, it is easy to see that, for all $a, b, c, d, e \in X$, $\langle\overrightarrow{a b}, \overrightarrow{a b}\rangle=d^{2}(a, b),\langle\overrightarrow{b a}, \overrightarrow{c d}\rangle=-\langle\overrightarrow{a b}, \overrightarrow{c d}\rangle,\langle\overrightarrow{a b}, \overrightarrow{c d}\rangle=\langle\overrightarrow{a e}, \overrightarrow{c d}\rangle+\langle\overrightarrow{e b}, \overrightarrow{c d}\rangle$. The space $X$ is said to satisfy the Cauchy Schwartz inequality if, for all $a, b, c, d \in X,\langle\overrightarrow{a b}, \overrightarrow{c d}\rangle \leq d(a, b) d(c, d)$. It is known (see [14]) that a geodesically connected metric space is a CAT(0) space if and only if it satisfies the Cauchy-Schwartz inequality.

In 2010, Kakavandi and Amini [15] introduced the concept of dual spaces in a complete CAT( 0$)$ space $X$ as follow. Let $C(X, \mathbb{R})$ be the space of all continuous real-valued functions on $X$. Consider a map $\Theta: \mathbb{R} \times X \times X \rightarrow C(X, \mathbb{R})$ defined by

$$
\Theta(t, a, b)(x)=t\langle\overrightarrow{a b}, \overrightarrow{a x}\rangle,(t \in \mathbb{R}, a, b, x \in X) .
$$

The Cauchy-Schwartz inequality implies that $\Theta(t, a, b)$ is a Lipschitz function with Lipschitz semi-norm $L(\Theta(t, a, b))=|t| d(a, b)(t \in \mathbb{R}, a, b \in X)$, where the Lipschitz semi-norm $L(\phi)$ of any function $\phi: X \rightarrow \mathbb{R}$ is given by

$$
L(\phi)=\sup \left\{\frac{\phi(x)-\phi(y)}{d(x, y)}: x, y \in X, x \neq y\right\} .
$$

A pseudometric $D$ on $\mathbb{R} \times X \times X$ is defined by

$$
D((t, a, b),(s, c, d))=L(\Theta(t, a, b)-\Theta(s, c, d)),
$$


for $t, s \in \mathbb{R}$ and $a, b, c, d \in X$. In a complete CAT(0) space, it was shown [15] that $D((t, a, b),(s, c$, $d))=0$ if and only if $t\langle\overrightarrow{a b}, \overrightarrow{x y}\rangle=s\langle\overrightarrow{c d}, \overrightarrow{x y}\rangle$, for all $x, y \in X$. Thus $D$ induces an equivalence relation on $\mathbb{R} \times X \times X$ with equivalence class defined by

$$
[t \overrightarrow{a b}]:=\{s \overrightarrow{s c d}: D((t, a, b),(s, c, d))=0\} .
$$

The pair $\left(X^{*}, D\right)$ is called the dual space of the metric space $(X, d)$, where $X^{*}=\{[t \overrightarrow{a b}]:(t, a, b) \in$ $\mathbb{R} \times X \times X\}$ and the function $D$ on $X^{*}$ is a metric.

In 2019, Cuntavepanit and Phuengrattana [16] studied the class of further generalized hybrid mappings [7] in Hadamard spaces. Very recently, Ali and Haruna [17] introduced a new class of mappings called further 2-generalized hybrid mappings in Hadamard spaces. This class of mappings contains further generalized hybrid and normally 2 generalized hybrid mappings as special cases.

Definition 1.2. Let $X$ be a complete CAT(0) space and $C$ be a nonempty subset of $X$. A mapping $T: C \rightarrow C$ is called further 2-generalized hybrid if there exist $\alpha_{1}, \alpha_{2}, \alpha_{3}, \beta_{1}, \beta_{2}, \beta_{3}, \varepsilon_{1}, \varepsilon_{2} \in \mathbb{R}$ such that (i) $\sum_{i=1}^{3}\left(\alpha_{i}+\beta_{i}\right) \geq 0, \varepsilon_{1}, \varepsilon_{2} \geq 0$, (ii) $\sum_{i=1}^{3} \alpha_{i}>0$ and

$$
\begin{gathered}
\text { (iii) } \alpha_{1} d^{2}\left(T^{2} x, T y\right)+\alpha_{2} d^{2}(T x, T y)+\alpha_{3} d^{2}(x, T y)+\beta_{1} d^{2}\left(T^{2} x, y\right) \\
+\beta_{2} d^{2}(T x, y)+\beta_{3} d^{2}(x, y)+\varepsilon_{1} d^{2}\left(x, T^{2} x\right)+\varepsilon_{2} d^{2}(x, T x) \leq 0 .
\end{gathered}
$$

for all $x, y \in C$.

Remark 1.1. If $\alpha_{1}=\beta_{1}=\varepsilon_{1}=0$, then the mapping reduces to further generalized hybrid in the sense of [16]. Also, the mapping reduces to normally 2-generalized hybrid mapping if $\varepsilon_{1}=\varepsilon_{2}=0$.

It is our purpose in this paper to construct a hybrid type iterative scheme that converges strongly to a common fixed point of further 2 generalized hybrid mappings in a flat Hadamard space. Our results improved and generalize the results in Takahashi, Wen and Yao [8] in the sense of the mapping and the space considered.

\section{PRELIMINARIES}

Throughout this paper, the symbols" $\rightarrow$ " and " $\rightarrow$ " represent the strong and $\Delta$-convergence, respectively. The following results play vital roles in establishing our main result.

Lemma 2.1. [13] Let $X$ be a $C A T(0)$ space and $x, y \in X, t \in[0,1]$. Then

(i) $d(z, t x \oplus(1-t) y) \leq t d(z, x)+(1-t) d(z, y)$;

(ii) $d^{2}(z, t x \oplus(1-t) y) \leq t d^{2}(z, x)+(1-t) d^{2}(z, y)-t(1-t) d^{2}(x, y)$.

The following Lemma can be deduced from Lemma 12 of [18] (see also [17]).

Lemma 2.2. Let $X$ be a complete $C A T(0)$ space and $x, y \in X, t_{i} \in(0,1)$. Then

$$
d^{2}\left(z, \oplus_{i=0}^{n} t_{i} x_{i}\right) \leq \sum_{i=0}^{n} t_{i} d^{2}\left(z, x_{i}\right)-t_{i} t_{j} d^{2}\left(x_{i}, x_{j}\right),
$$

where $i, j \in\{0,1, \ldots, n\}$ and $\sum_{i=0}^{n} t_{i}=1$

Lemma 2.3. [19] Let $X$ be a complete $C A T(0)$ space. Then every bounded sequence in $X$ has a $\Delta$-convergence subsequence. 
Lemma 2.4. [20] Let $X$ be a complete $C A T(0)$ space. Let $\left\{x_{n}\right\}$ be a sequence in $X$ and $x \in X$. Then $\left\{x_{n}\right\} \Delta$-converges to $x$ if and only if $\limsup _{n \rightarrow \infty}\left\langle\overrightarrow{x_{n} x}, \overrightarrow{y x}\right\rangle \leq 0, \forall y \in X$.

\section{MAIN RESUlTS}

In this section, $X$ is considered to be a flat Hadamard space. We then construct a hybrid type iterative scheme that converges strongly to a common fixed point of further 2 generalized hybrid mappings in the space.

Lemma 3.1. Let $C$ be a nonempty subset of a flat Hadamard space $X$, and let $T: C \rightarrow C$ be a further 2-generalized hybrid mapping. Let $\left\{x_{n}\right\}$ be a bounded sequence in $C$ such that $\Delta-$ $\lim _{n \rightarrow \infty} x_{n}=z$ and $d\left(x_{n}, T x_{n}\right) \rightarrow 0, d\left(x_{n}, T^{2} x_{n}\right) \rightarrow 0$ as $n \rightarrow \infty$. Then $z \in F(T)$.

Proof. Let $\left\{x_{n}\right\} \subset C$ be a sequence such that $x_{n} \rightarrow z,\left(x_{n}-T x_{n}\right) \rightarrow 0$ and $\left(x_{n}-T^{2} x_{n}\right) \rightarrow 0$ as $n \rightarrow \infty$. Note that $T: C \rightarrow C$ is a generic 2-generalized Bregman nonspreading mapping. Following the similar techniques as in [17, Lemma 3.1], we get $d(z, T y) \leq d(z, y)$ for all $y \in C$. Setting $y=z$, we obtain $d(z, T z)=0$. Thus, $z \in F(T)$. This completes the proof.

Lemma 3.2. Let $C$ be a nonempty subset of a flat Hadamard space $X$, and let $T: C \rightarrow C$ be a further 2-generalized hybrid mapping. If $F(T) \neq \emptyset$, then $T$ is quasi-nonexpansive.

Proof. Let $T$ be a further 2-generalized hybrid mapping with $F(T) \neq \emptyset$. In particular, let $x \in$ $F(T)$ such that $T^{2} x=T x=x$. Now, from the definition of $T$, we get

$$
\begin{aligned}
\alpha_{1} d^{2}(x, T y) & +\alpha_{2} d^{2}(x, T y)+\alpha_{3} d^{2}(x, T y)+\beta_{1} d^{2}(x, y) \\
& +\beta_{2} d^{2}(x, y)+\beta_{3} d^{2}(x, y)+\varepsilon_{1} d^{2}(x, x)+\varepsilon_{2} d^{2}(x, x) \leq 0 .
\end{aligned}
$$

for all $y \in C$. This implies

$$
\left(\alpha_{1}+\alpha_{2}+\alpha_{3}\right) d^{2}(x, T y) \leq-\left(\beta_{1}+\beta_{2}+\beta_{3}\right) d^{2}(x, y),
$$

for all $y \in C$. Since $\sum_{i=1}^{3} \alpha_{i}>0$, we obtain

$$
d^{2}(x, T y) \leq-\frac{\left(\beta_{1}+\beta_{2}+\beta_{3}\right)}{\left(\alpha_{1}+\alpha_{2}+\alpha_{3}\right)} d^{2}(x, y),
$$

for all $y \in C$. From the fact that $\sum_{i=1}^{3}\left(\alpha_{i}+\beta_{i}\right) \geq 0$, we have

$$
d^{2}(x, T y) \leq d^{2}(x, y) \text {. }
$$

Thus, $d(x, T y) \leq d(x, y)$ for all $y \in C$. Hence $T$ is quasi-nonexpansive. This completes the prove.

Theorem 3.1. Let $C$ be a nonempty, convex subset of a flat Hadamard space $X$ which satisfies the $(\mathbb{S})$ property and the $\left(\bar{Q}_{4}\right)$ condition. Let $S, T: C \rightarrow C$ be further 2-generalized hybrid mappings such that $\mathscr{F}=F(S) \cap F(T) \neq \emptyset$. Let $\left\{x_{n}\right\} \subset C$ be a sequence generated by $x_{1} \in C$

$$
\left\{\begin{array}{l}
\left.y_{n}=\alpha_{n} x_{n} \oplus \beta_{n}\left(\delta_{n} S x_{n} \oplus\left(1-\delta_{n}\right) T x_{n}\right) \oplus \gamma_{n}\left(\lambda_{n} S^{2} x_{n} \oplus\left(1-\lambda_{n}\right) T^{2} x_{n}\right)\right), \\
C_{n}=\left\{p \in C: d\left(p, y_{n}\right) \leq d\left(p, x_{n}\right)\right\}, \\
Q_{n}=\left\{p \in C:\left\langle\overrightarrow{x_{1} x_{n}}, \overrightarrow{x_{n} \vec{p}}\right\rangle \geq 0\right\}, \\
x_{n+1}=P_{C_{n} \cap Q_{n}}\left(x_{1}\right), \quad n \in \mathbb{N},
\end{array}\right.
$$

where $\left\{\alpha_{n}\right\},\left\{\beta_{n}\right\},\left\{\gamma_{n}\right\},\left\{\delta_{n}\right\},\left\{\lambda_{n}\right\} \subset[a, b] \subset(0,1)$ and $\alpha_{n}+\beta_{n}+\gamma_{n}=1$. Then $\left\{x_{n}\right\}$ converges strongly to $z=P_{\mathscr{F}}\left(x_{1}\right)$. 
Proof. Note that $\mathscr{F}$ is closed and convex and hence $z=\mathrm{P}_{\mathscr{F}}\left(x_{1}\right)$ is well defined. From the definition of $C_{n}, d\left(p, y_{n}\right) \leq d\left(p, x_{n}\right)$ if and only if $-d^{2}\left(x_{n}, y_{n}\right)+\left\langle\overrightarrow{p y_{n}}, \overrightarrow{x_{n} y_{n}}\right\rangle \leq 0$. Thus, it is easy to see that both $C_{n}, Q_{n}$ and $C_{n} \cap Q_{n}$ are closed and convex. For the closedness, let $p_{k} \in C_{n}$ be such that $p_{k} \rightarrow p$ as $k \rightarrow \infty$. Then, we show $p \in C_{n}$. But

$$
\begin{aligned}
& -d^{2}\left(x_{n}, y_{n}\right)+\left\langle\overrightarrow{p y_{n}}, \overrightarrow{x_{n} y_{n}}\right\rangle \\
& =-d^{2}\left(x_{n}, y_{n}\right)+\left\langle\overrightarrow{\lim _{k \rightarrow \infty} p_{k} y_{n}}, \overrightarrow{x_{n} y_{n}}\right\rangle \\
& =-d^{2}\left(x_{n}, y_{n}\right)+\lim _{k \rightarrow \infty}\left\langle\overrightarrow{p_{k} y_{n}}, \overrightarrow{x_{n} y_{n}}\right\rangle \\
& =\lim _{k \rightarrow \infty}\left(-d^{2}\left(x_{n}, y_{n}\right)+\left\langle\overrightarrow{p_{k} y_{n}}, \overrightarrow{x_{n} y_{n}}\right\rangle\right) \leq 0 .
\end{aligned}
$$

Thus, $C_{n}$ is closed. For the convexity, let $p_{1}, p_{2} \in C_{n}$. Then, we show that $p=t p_{1} \oplus(1-t) p_{2} \in$ $C_{n}$ for $t \in[0,1]$. Using equation (1.2), we get

$$
\begin{aligned}
& -d^{2}\left(x_{n}, y_{n}\right)+\left\langle\overrightarrow{p y_{n}}, \overrightarrow{x_{n} y_{n}}\right\rangle \\
& =-d^{2}\left(x_{n}, y_{n}\right)+\left\langle\overrightarrow{\left(t p_{1} \oplus(1-t) p_{2}\right) y_{n}}, \overrightarrow{x_{n} y_{n}}\right\rangle \\
& =-d^{2}\left(x_{n}, y_{n}\right)+t\left\langle\overrightarrow{p_{1} y_{n}}, \overrightarrow{x_{n} y_{n}}\right\rangle+(1-t)\left\langle\overrightarrow{p_{2} y_{n}}, \overrightarrow{x_{n} y_{n}}\right\rangle \leq 0 \text {. }
\end{aligned}
$$

Thus, $C_{n}$ is convex. Therefore, $C_{n}$ is closed and convex. Following similar techniques, we see that $Q_{n}$ is closed and convex. Hence $C_{n} \cap Q_{n}$ is closed and convex.

Let $u_{n}=\delta_{n} S x_{n} \oplus\left(1-\delta_{n}\right) T x_{n}$ and $v_{n}=\lambda_{n} S^{2} x_{n} \oplus\left(1-\lambda_{n}\right) T^{2} x_{n}$. Let $z \in \mathscr{F}$. Using Lemma 2.1 , we get

$$
\begin{aligned}
d\left(z, u_{n}\right) & =d\left(z, \delta_{n} S x_{n} \oplus\left(1-\delta_{n}\right) T x_{n}\right) \\
& \leq \delta_{n} d\left(z, S x_{n}\right)+\left(1-\delta_{n}\right) d\left(z, T x_{n}\right) \\
& =d\left(z, x_{n}\right) .
\end{aligned}
$$

Similarly,

$$
\begin{aligned}
d\left(z, v_{n}\right) & =d\left(z, \lambda_{n} S^{2} x_{n} \oplus\left(1-\lambda_{n}\right) T^{2} x_{n}\right) \\
& \leq \lambda_{n} d\left(z, S^{2} x_{n}\right)+\left(1-\lambda_{n}\right) d\left(z, T^{2} x_{n}\right) \\
& \leq \lambda_{n} d\left(z, S x_{n}\right)+\left(1-\lambda_{n}\right) d\left(z, T x_{n}\right) \\
& =d\left(z, x_{n}\right) .
\end{aligned}
$$

It follows that

$$
\begin{aligned}
d\left(z, y_{n}\right) & =d\left(z, \alpha_{n} x_{n} \oplus \beta_{n} u_{n} \oplus \gamma_{n} v_{n}\right) \\
& \leq \alpha_{n} d\left(z, x_{n}\right)+\beta_{n} d\left(z, u_{n}\right)+\gamma_{n} d\left(z, v_{n}\right) \\
& \leq \alpha_{n} d\left(z, x_{n}\right)+\beta_{n} d\left(z, x_{n}\right)+\gamma_{n} d\left(z, x_{n}\right) \\
& =d\left(z, x_{n}\right) .
\end{aligned}
$$

Therefore, $z \in C_{n}$ and hence $\mathscr{F} \in C_{n}$. We can now use induction to show that $\mathscr{F} \in Q_{n} \cap C_{n}$, $\forall n \in \mathbb{N}$. For $n=1$, we see that $\mathscr{F} \subset C_{1} \cap Q_{1}$ since $\mathscr{F} \subset C_{1}$ and $Q_{1}=C$. Suppose, for some $k \geq 1, \mathscr{F} \subset C_{k} \cap Q_{k}$. Using $x_{k+1}=P_{C_{k} \cap Q_{k}} x_{1}$ and the property of metric projections, we have

$$
\left\langle\overrightarrow{x_{k+1} x_{1}}, \overrightarrow{p x_{k+1}}\right\rangle \geq 0
$$


for all $p \in C_{k} \cap Q_{k}$. Since $\mathscr{F} \subset C_{k} \cap Q_{k}$, we have

$$
\left\langle\overrightarrow{x_{k+1} x_{1}}, \overrightarrow{z x_{k+1}}\right\rangle \geq 0
$$

for all $z \in \mathscr{F}$. This implies $\mathscr{F} \subset C_{k+1} \cap Q_{k+1}$ and therefore $\mathscr{F} \subset C_{n} \cap Q_{n} \forall n \in \mathbb{N}$. Hence, the sequence $\left\{x_{n}\right\}$ is well defined.

We next show that the sequence $\left\{x_{n}\right\}$ is bounded. We know from the definition of $Q_{n}$ that $x_{n}=P_{Q_{n}} x_{1}$. Thus, using the property of metric projection, we get

$$
\begin{aligned}
d\left(x_{n}, x_{1}\right) & =d\left(P_{Q_{n}} x_{1}, x_{1}\right) \\
& \leq d\left(z, x_{1}\right)-d\left(z, P_{Q_{n}} x_{1}\right) \\
& =d\left(z, x_{1}\right) .
\end{aligned}
$$

Thus, the sequence $\left\{d\left(x_{n}, x_{1}\right)\right\}$ is bounded, so is $\left\{x_{n}\right\}$. In view of $x_{n}=P_{Q_{n}} x_{1}$ and $x_{n+1} \in Q_{n}$, we have

$$
\begin{aligned}
0 & \leq\left\langle\overrightarrow{x_{1} x_{n}}, \overrightarrow{x_{n} x_{n+1}}\right\rangle \\
& =d^{2}\left(x_{1}, x_{n+1}\right)+d^{2}\left(x_{n}, x_{n}\right)-d^{2}\left(x_{1}, x_{n}\right)-d^{2}\left(x_{n}, x_{n+1}\right) \\
& \leq d^{2}\left(x_{1}, x_{n+1}\right)-d^{2}\left(x_{1}, x_{n}\right) .
\end{aligned}
$$

This implies $d\left(x_{1}, x_{n}\right) \leq d\left(x_{1}, x_{n+1}\right)$. Thus, $\left\{d\left(x_{1}, x_{n}\right)\right\}$ is monotone increasing. Since it is bounded, then $\lim _{n \rightarrow \infty} d\left(x_{n}, x_{1}\right)$ exists. From equation (3.2), we see that

$$
d^{2}\left(x_{n}, x_{n+1}\right) \leq d^{2}\left(x_{1}, x_{n+1}\right)-d^{2}\left(x_{1}, x_{n}\right) .
$$

From $x_{n+1} \in C_{n}$, we obtain

$$
d\left(x_{n+1}, y_{n}\right) \leq d\left(x_{n+1}, x_{n}\right) .
$$

From the property of metric distances, we get that

$$
d\left(x_{n}, y_{n}\right) \leq d\left(x_{n}, x_{n+1}\right)+d\left(x_{n+1}, y_{n}\right) .
$$

From the existence of $\lim _{n \rightarrow \infty} d\left(x_{n}, x_{1}\right),(3.3),(3.4)$ and (3.5), we get that

$$
\lim _{n \rightarrow \infty} d\left(x_{n}, x_{n+1}\right)=0, \lim _{n \rightarrow \infty} d\left(x_{n+1}, y_{n}\right)=0, \lim _{n \rightarrow \infty} d\left(x_{n}, y_{n}\right)=0 .
$$

On the other hand, using Lemma 2.2, we have

$$
\begin{aligned}
d^{2}\left(z, y_{n}\right) & =d^{2}\left(z, \alpha_{n} x_{n} \oplus \beta_{n} u_{n} \oplus \gamma_{n} v_{n}\right) \\
& \leq \alpha_{n} d^{2}\left(z, x_{n}\right)+\beta_{n} d^{2}\left(z, u_{n}\right)+\gamma_{n} d^{2}\left(z, v_{n}\right)-\alpha_{n} \beta_{n} d^{2}\left(x_{n}, u_{n}\right) \\
& \leq \alpha_{n} d^{2}\left(z, x_{n}\right)+\beta_{n} d^{2}\left(z, x_{n}\right)+\gamma_{n} d^{2}\left(z, x_{n}\right)-\alpha_{n} \beta_{n} d^{2}\left(x_{n}, u_{n}\right) \\
& =d^{2}\left(z, x_{n}\right)-\alpha_{n} \beta_{n} d^{2}\left(x_{n}, u_{n}\right) .
\end{aligned}
$$

Thus,

$$
d^{2}\left(z, y_{n}\right) \leq d^{2}\left(z, x_{n}\right)-\alpha_{n} \beta_{n} d^{2}\left(x_{n}, u_{n}\right)
$$

Similarly, one has

$$
d^{2}\left(z, y_{n}\right) \leq d^{2}\left(z, x_{n}\right)-\alpha_{n} \gamma_{n} d^{2}\left(x_{n}, v_{n}\right) .
$$

It follows from (3.7) and (3.8) that

$$
\alpha_{n} \beta_{n} d^{2}\left(x_{n}, u_{n}\right) \leq d^{2}\left(z, x_{n}\right)-d^{2}\left(z, y_{n}\right)
$$


and

$$
\alpha_{n} \gamma_{n} d^{2}\left(x_{n}, v_{n}\right) \leq d^{2}\left(z, x_{n}\right)-d^{2}\left(z, y_{n}\right) .
$$

Using the quasilinearization definition, the Cauchy-Schwartz inequality, (3.6) and (3.9), we conclude that

$$
\begin{aligned}
\alpha_{n} \beta_{n} d^{2}\left(x_{n}, u_{n}\right) & \leq d^{2}\left(z, x_{n}\right)-d^{2}\left(z, y_{n}\right) \\
& \leq d^{2}\left(x_{n}, x_{n}\right)-d^{2}\left(x_{n}, y_{n}\right)+2\left\langle\overrightarrow{z x_{n}}, \overrightarrow{y_{n} x_{n}}\right\rangle \\
& \leq 2\left\langle\overrightarrow{z x_{n}}, \overrightarrow{y_{n} x_{n}}\right\rangle \\
& \leq 2 d\left(z, x_{n}\right) d\left(y_{n}, x_{n}\right) \rightarrow 0 \text { as } n \rightarrow \infty .
\end{aligned}
$$

Similarly, it follows from (3.10) that

$$
\alpha_{n} \gamma_{n} d^{2}\left(x_{n}, v_{n}\right) \leq 2 d\left(z, x_{n}\right) d\left(y_{n}, x_{n}\right) \rightarrow 0 \text { as } n \rightarrow \infty .
$$

Using the fact that $\alpha_{n}, \beta_{n}, \gamma_{n} \subset[a, b] \subset(0,1)$, we conclude that

$$
d\left(x_{n}, u_{n}\right) \rightarrow 0, d\left(x_{n}, v_{n}\right) \rightarrow 0 \text { as } n \rightarrow \infty
$$

and

$$
\begin{aligned}
d^{2}\left(z, u_{n}\right) & =d^{2}\left(z, \delta_{n} S x_{n} \oplus\left(1-\delta_{n}\right) T x_{n}\right) \\
& \leq \delta_{n} d^{2}\left(z, S x_{n}\right)+\left(1-\delta_{n}\right) d^{2}\left(z, T x_{n}\right)-\delta_{n}\left(1-\delta_{n}\right) d^{2}\left(S x_{n}, T x_{n}\right) \\
& \leq d^{2}\left(z, x_{n}\right)-\delta_{n}\left(1-\delta_{n}\right) d^{2}\left(S x_{n}, T x_{n}\right)
\end{aligned}
$$

Thus,

$$
\delta_{n}\left(1-\delta_{n}\right) d^{2}\left(S x_{n}, T x_{n}\right) \leq d^{2}\left(z, x_{n}\right)-d^{2}\left(z, u_{n}\right)
$$

Similarly, one has

$$
\lambda_{n}\left(1-\lambda_{n}\right) d^{2}\left(S^{2} x_{n}, T^{2} x_{n}\right) \leq d^{2}\left(z, x_{n}\right)-d^{2}\left(z, v_{n}\right) .
$$

Applying the quasilinearization property, the Cauchy-Schwartz inequality, (3.11), (3.12), we get

$$
\begin{aligned}
\delta_{n}\left(1-\delta_{n}\right) d^{2}\left(S x_{n}, T x_{n}\right) & \leq d^{2}\left(z, x_{n}\right)-d^{2}\left(z, u_{n}\right) \\
& \leq d^{2}\left(x_{n}, x_{n}\right)-d^{2}\left(x_{n}, u_{n}\right)+2\left\langle\overrightarrow{z x_{n}}, \overrightarrow{u_{n} x_{n}}\right\rangle \\
& \leq 2\left\langle\overrightarrow{z x_{n}}, \overrightarrow{u_{n} x_{n}}\right\rangle \\
& \leq 2 d\left(z, x_{n}\right) d\left(u_{n}, x_{n}\right) \rightarrow 0 \text { as } n \rightarrow \infty .
\end{aligned}
$$

Similarly, one has

$$
\lambda_{n}\left(1-\lambda_{n}\right) d^{2}\left(S^{2} x_{n}, T^{2} x_{n}\right) \leq 2 d\left(z, x_{n}\right) d\left(v_{n}, x_{n}\right) \rightarrow 0 \text { as } n \rightarrow \infty .
$$

Using the fact that $\delta_{n}, \lambda_{n} \subset[a, b] \subset(0,1)$, we conclude that

$$
d\left(S x_{n}, T x_{n}\right) \rightarrow 0, d\left(S^{2} x_{n}, T^{2} x_{n}\right) \rightarrow 0 \text { as } n \rightarrow \infty .
$$

Now,

$$
\begin{aligned}
d\left(x_{n}, T x_{n}\right) & \leq d\left(x_{n}, u_{n}\right)+d\left(u_{n}, T x_{n}\right) \\
& \leq d\left(x_{n}, u_{n}\right)+\delta_{n} d\left(S x_{n}, T x_{n}\right)+\left(1-\delta_{n}\right) d\left(T x_{n}, T x_{n}\right)
\end{aligned}
$$


and

$$
\begin{aligned}
d\left(x_{n}, T^{2} x_{n}\right) & \leq d\left(x_{n}, v_{n}\right)+d\left(v_{n}, T^{2} x_{n}\right) \\
& \leq d\left(x_{n}, v_{n}\right)+\lambda_{n} d\left(S^{2} x_{n}, T^{2} x_{n}\right)+\left(1-\lambda_{n}\right) d\left(T^{2} x_{n}, T^{2} x_{n}\right) .
\end{aligned}
$$

Using (3.14), (3.15) and (3.16), we get

$$
\lim _{n \rightarrow \infty} d\left(x_{n}, T x_{n}\right)=0 \text { and } \lim _{n \rightarrow \infty} d\left(x_{n}, T^{2} x_{n}\right)=0 .
$$

But

$$
d\left(x_{n}, S x_{n}\right) \leq d\left(x_{n}, T x_{n}\right)+d\left(T x_{n}, S x_{n}\right)
$$

and

$$
d\left(x_{n}, S^{2} x_{n}\right) \leq d\left(x_{n}, T^{2} x_{n}\right)+d\left(T^{2} x_{n}, S^{2} x_{n}\right) .
$$

Using (3.14) and (3.17) in (3.18) and (3.19), we get

$$
\lim _{n \rightarrow \infty} d\left(x_{n}, S x_{n}\right)=0 \text { and } \lim _{n \rightarrow \infty} d\left(x_{n}, S^{2} x_{n}\right)=0 .
$$

Since $X$ is a complete CAT(0) space and the sequence $\left\{x_{n}\right\}$ is bounded, we conclude from Lemma 2.3 that there exists a subsequence $\left\{x_{n_{k}}\right\}$ of $\left\{x_{n}\right\}$ such that $\Delta-\lim _{k \rightarrow \infty} x_{n_{k}}=u$. Using Lemma 3.1, (3.17) and (3.20), we conclude that $u \in \mathscr{F}$.

Now, let $v=P_{\mathscr{F}} x_{1}$. Since $x_{n+1}=P_{C_{n} \cap Q_{n}} x_{1}$ and $v \in C_{n} \cap Q_{n}$, then $d\left(x_{n+1}, x_{1}\right) \leq d\left(v, x_{1}\right)$. Also, $x_{n_{k}} \rightarrow u$ and $d(.,$.$) is convex and lower semicontinuous (hence, \Delta$-lower semicontinuous), we get

$$
d\left(u, x_{1}\right) \leq \liminf _{k \rightarrow \infty} d\left(x_{n_{k}}, x_{1}\right) \leq d\left(v, x_{1}\right) .
$$

From the definition of $v$, we can conclude that $u=v$. So, $x_{n} \rightarrow v$. Using the definition of the quasilinearization and Lemma 2.4, we get

$$
\begin{aligned}
\limsup _{n \rightarrow \infty} d^{2}\left(x_{n}, v\right) & =\limsup _{n \rightarrow \infty}\left(d^{2}\left(v, x_{1}\right)+d^{2}\left(x_{n}, x_{1}\right)-2\left\langle\overrightarrow{v x_{1}}, \overrightarrow{x_{n} x_{1}}\right\rangle\right) \\
& \leq \underset{n \rightarrow \infty}{\limsup }\left(d^{2}\left(v, x_{1}\right)+d^{2}\left(v, x_{1}\right)-2\left\langle\overrightarrow{v x_{1}}, \overrightarrow{x_{n} x_{1}}\right\rangle\right) \\
& =2 \limsup _{n \rightarrow \infty}\left(d^{2}\left(v, x_{1}\right)-\left\langle\overrightarrow{v x_{1}}, \overrightarrow{x_{n} x_{1}}\right\rangle\right) \\
& =2 \limsup _{n \rightarrow \infty}\left(\left\langle\overrightarrow{v x_{1}}, \overrightarrow{v x_{1}}\right\rangle+\left\langle\overrightarrow{v x_{1}}, \overrightarrow{x_{1} x_{n}}\right\rangle\right) \\
& =2 \limsup _{n \rightarrow \infty}\left\langle\overrightarrow{v x_{1}}, \overrightarrow{v x_{n}}\right\rangle \leq 0 .
\end{aligned}
$$

Thus $\lim _{n \rightarrow \infty} d^{2}\left(x_{n}, v\right)=0$. Therefore, $x_{n} \rightarrow v$. This completes the proof.

In view of Remark 1.1, we have the following results as corollaries of Theorem 3.1.

Corollary 3.1. Let $C$ be a nonempty, convex subset of a flat Hadamard space $X$ which satisfies the $(\mathbb{S})$ property and the $\left(\bar{Q}_{4}\right)$ condition. Let $S, T: C \rightarrow C$ be normally 2-generalized hybrid mappings such that $\mathscr{F}=F(S) \cap F(T) \neq \emptyset$. Let $\left\{x_{n}\right\}$ be a sequence defined by (3.1). Then $\left\{x_{n}\right\}$ converges strongly to $z=P_{\mathscr{F}}\left(x_{1}\right)$.

Proof. Since further 2-generalized hybrid mapping is reduced to normally 2-generalized hybrid mappings if $\varepsilon_{1}=\varepsilon_{2}=0$. It follows from Theorem 3.1 that $\left\{x_{n}\right\}$ converges strongly to $z=$ $\mathrm{P}_{\mathscr{F}}\left(x_{1}\right)$. This completes the proof. 
Corollary 3.2. Let $C$ be a nonempty, convex subset of a flat Hadamard space $X$ which satisfies the $(\mathbb{S})$ property and the $\left(\bar{Q}_{4}\right)$ condition. Let $S, T: C \rightarrow C$ be further generalized hybrid mappings such that $\mathscr{F}=F(S) \cap F(T) \neq \emptyset$. Let $\left\{x_{n}\right\}$ be a sequence defined by (3.1). Then $\left\{x_{n}\right\}$ converges strongly to $z=P_{\mathscr{F}}\left(x_{1}\right)$.

Proof. If $\alpha_{1}=\beta_{1}=\varepsilon_{1}=0$, i.e., a further 2-generalized hybrid mapping is reduced to a further generalized hybrid mapping, then we see from Theorem 3.1 that $\left\{x_{n}\right\}$ converges strongly to $z=\mathrm{P}_{\mathscr{F}}\left(x_{1}\right)$. This completes the proof.

In view of the fact that Hilbert spaces are Hadamard spaces, the following result can easily be deduced from Corollary 3.1.

Corollary 3.3. [8, Theorem 3.1] Let $C$ be a nonempty, closed and convex subset of a Hilbert space $X$. Let $S, T: C \rightarrow C$ be normally 2-generalized hybrid mappings such that $\mathscr{F}=F(S) \cap$ $F(T) \neq \emptyset$. Let $\left\{x_{n}\right\} \subset C$ be a sequence generated by

$$
\left\{\begin{array}{l}
x_{1} \in C, \\
y_{n}=\alpha_{n} x_{n}+\beta_{n}\left(\delta_{n} S+\left(1-\delta_{n}\right) T\right) x_{n}+\gamma_{n}\left(\lambda_{n} S^{2}+\left(1-\lambda_{n}\right) T^{2}\right) x_{n}, \\
C_{n}=\left\{z \in C:\left\|y_{n}-z\right\| \leq\left\|x_{n}-z\right\|\right\} \\
Q_{n}=\left\{z \in C:\left\langle x_{n}-z, x_{1}-x_{n}\right\rangle \geq 0\right\} \\
x_{n+1}=P_{C_{n} \cap Q_{n}} x_{1}, \quad \forall n \in \mathbb{N},
\end{array}\right.
$$

where $\left\{\alpha_{n}\right\},\left\{\beta_{n}\right\},\left\{\gamma_{n}\right\},\left\{\delta_{n}\right\},\left\{\lambda_{n}\right\} \subset[a, b] \subset(0,1)$ and $\alpha_{n}+\beta_{n}+\gamma_{n}=1$. Then $\left\{x_{n}\right\}$ converges strongly to $z=P_{\mathscr{F}}\left(x_{1}\right)$.

Proof. Since the normally 2-generalized hybrid mapping defined in Hadamard spaces reduces to the one introduced by Kondo and Takahashi [6] in Hilbert spaces, we obtain from Corollary 3.1 the desired conclusion immediately.

\section{REFERENCES}

[1] P. Kocourek, W. Takahashi, J.-C. Yao, Fixed points and weak convergence theorems for generalized hybrid mappings in Hilbert spaces, Taiwanese J. Math. 14 (2010), 2497-2511.

[2] F. Kohsaka, W. Takahashi, Fixed point theorems for a class of nonlinear mappings related to a maximal monotone operators in Banach spaces, Arch. Math. (Basel), 19 (2008), 166-177.

[3] W. Takahashi, Fixed point theorems for new nonlinear mappings in Hilbert spaces, J. Nonlinear Convex Anal. 11 (2010), 79-88.

[4] F. Kohsaka, W. Takahashi, Existence and approximation of firmly nonexpansive type mappings in Banach spaces, SIAM J. Optim. 19 (2008), 824-835.

[5] W. Takahashi, N.-C. Wong, J.-C. Yao, Attractive point and weak convergence theorem for new generalized hybrid mappings in Hilbert spaces, J. Nonlinear Convex Anal. 13 (2012), 745-757.

[6] A. Kondo, W. Takahashi, Attractive point and weak convergence theorems normally n-generalized hybrid mappings in Hilbert spaces, Linear Nonlinear Anal. 3 (2017), 297-310.

[7] S. H. Khan, Iterative approximations of common attractive points of further generalized hybrid mappings, Fixed Point Theory Appl. 2018 (2018), 69.

[8] W. Takahashi, C.-F. Wen, J.-C. Yao, Strong convergence theorems by hybrid methods for nonommutative normally 2-generalized hybrid mappings in Hilbert spaces, Appl. Anal. Optim. 3 (2019), 43-56.

[9] M. Bridson, A. Haefliger, A Metric Space of Non-Positive Curvature, Springer, Berlin, 1999.

[10] K. Goebel, S. Reich, Uniform Convexity, Hyperbolic Geometry and Nonexpansive Mappings, Marcel Dekker, New York, 1984.

[11] S. Reich, I. Shafrir, Nonexpansive iterations in hyperbolic spaces, Nonlinear Anal. 15 (1990), 537-558. 
[12] H. Khatibzadeh, V. Mohebbi, Monotone and pseudomonotone problems in Hadamard spaces, arXiv:1611.01829v1 [math.FA], 2016.

[13] S. Dhompongsa, B. Panyanak, On $\Delta$-convergence theorems in CAT(0) spaces, Comput. Math. Appl. 56 (2008), 2572-2579.

[14] I.D. Berg, I.G. Nicolaev, Quasilinearization and curvature of Alexandrov spaces, Geom. Dedicata, 133 (2008), 195-218.

[15] B.A. Kakavandi, M. Amini, Duality and subdifferential for convex functions on complete CAT(0) metric spaces, Nonlinear Anal. 73 (2010), 3450-3455.

[16] A. Cuntavepanit, W. Phuengrattana, Iterative approximation of attractive points of further generalized hybrid mappings in Hadamard spaces, Fixed Point Theory Appl. 2019 (2019), 3.

[17] B. Ali, L.Y. Haruna, Attractive points of further 2-generalized hybrid mapping in a complete CAT(0) space, J. Nonlinear Var. Anal. 3 (2019), 235-246.

[18] C.E. Chidume, A.U. Bello, P. Ndambomve, Strong and $\Delta$-convergence theorems for common fixed points of a finite family of multivalued demicontractive mappings in CAT(0) spaces, Abstr. Appl. Anal. 2014 (2014), 805168.

[19] W.A. Kirk, B. Panyanak, A concept of convergence in geodesic spaces, Nonlinear Anal. 68 (2008), 36893696.

[20] B.A. Kakavandi, Weak topologies in complte CAT(0) metric spaces, Proc. Amer. Math. Soc. 141 (2013), 1029-1039. 